\title{
Stochastic Analysis and Reliability-Cost Optimization of Distributed Generators and Air Source Heat Pumps
}

\author{
Roberto Rocchetta \\ School of Engineering \\ Institute for Risk and Uncertainty \\ Liverpool University \\ Email: roberto.rocchetta@liverpool.ac.uk
}

\author{
Edoardo Patelli \\ School of Engineering \\ Institute for Risk and Uncertainty \\ Liverpool University \\ Email: edoardo.patelli@liverpool.ac.uk
}

\begin{abstract}
This paper presents a framework for stochastic analysis, simulation and optimisation of electric power grids combined with heat district networks. In this framework, distributed energy sources (e.g. wind turbines and storage systems) can be integrated within the grids and their performance is modelled. The effect of variable weather conditions on the overall system cost and reliability is computed taking into account relevant sources of uncertainty. A Monte Carlo Optimal Power Flow simulator is employed and statistical indicators of the system cost and reliability are computed. Reliability and cost expectations are used to compare 4 different investments on heat pumps and electric power generators to be installed on a real-world grid. Generators' sizes and positions are analysed to reveal the sensitivity of the cost and reliability of the grid and an optimal investment problem is tackled by using a multi-objective genetic algorithm.
\end{abstract}

Keyworkds- Renewable Power; Interconnected Grids Reliability; Heat Pumps; Sensitivity; Stochastic Optimisation Analysis;

\section{INTRODUCTION}

Future grids should provide reliable power supply at lower achievable cost, mitigating power losses and overall negative environmental impact. The present view on Smart Grid projects (e.g. [1]) generally rates the power grid as the most prominent infrastructure whilst different systems (e.g. transportation and heat district networks) received a relatively limited consideration. In the last years, however, several researchers pointed out the benefits of combining analysis of power grids to different networked systems which are inevitably linked. For instance, integrated analysis of interconnected heat and power networks [2]- [3]- [4] can provide better insights on systems collective behaviours and interactions. Similar conclusions can be obtained by analysis of the so-called multi-energy-systems (i.e. systems for which electricity, heating, cooling, fuels, transport optimally interact with each other at various levels [5]).

Distributed Generators (DGs) and renewable energy sources are broadly integrated within power girds and, if optimally allocated and operated, can reduce electric energy losses, minimise carbon dioxide emission and improve grid reliability [6]- [7]. One of the most discussed issues of renewable generators is the uncertainty associated with their power outputs [8]- [9]- [10], which generally discourages investments on high energy penetrations. In fact, renewable power availability is generally hard to predict because depends on variable and uncertain weather conditions. To deal with this issue, robust stochastic frameworks specifically designed to deal with the inevitable uncertainty associated with renewable technologies, have been proposed [6]- [11][12]- [13].

Efficient generation of heat and modern heat distribution technologies (such as heat pumps and heat district networks) are valuable resources for future grid sustainability. As a matter of fact, the overall buildings energy consumption shares a large amount of total energy demand of developed countries (up to 40\%) [14]. It is therefore of paramount importance for the future grid sustainability to minimise the overall buildings' energy consumption and at the same time maximise energy efficiency and heat supply reliability. For this purpose, buildings envelopes can be enhanced and efficient heating and cooling systems installed (e.g. exploiting renewable air and ground energy [15]- [16]- [17]). If energy-efficient technologies are employed, air source heat pumps are some of the most interesting options available on the market [15].

Air source Heat Pumps (HPs) are systems which transfer heat from inside to outside a building (cooling) or from outside to inside a building (heating) by exploiting a refrigeration cycle. Air source heat pumps are relatively convenient economically speaking and generally achieve high coefficients of performance (i.e. efficiency). One of the major downsides is that their operative states are strongly affected by uncertain, variable ambient air temperate and, thus, some sort of heating back-up system is normally required to guarantee a reliable heating supply [18]. An increasing number of research papers discussed the effects of combining 
heat pumps with renewable distributed electric generators [19]- [20]. However, to the author's knowledge, just a few works embedded distributed air source heat pumps model within stochastic frameworks for uncertainty quantification (e.g. [21]- [22]). For instance, Essa and Cipcigan [21] proposed a stochastic analysis and integration of Electric Vehicles, Air Source Heat Pumps, and photovoltaic panels in a low voltage grid. Cesena, Capuder and Mancarella [22] proposed an optimisation framework for distributed multienergy-systems allocating combined heat power devices and considering long-term uncertainty. More research should be focused on improving and developing stochastic frameworks for combined multi-energy-systems analysis and, to the authors' opinion, particular efforts should be devoted to the minimization of costs while, at the same time, maximising system sustainability and reliability.

This paper presents a computational framework for stochastic simulation and optimisation of distributed generators within interconnected heat and electric power grids. The considered electric and heat power generators can be allocated within the grids' nodes. The renewable power outputs are affected by uncertain weather conditions. Relevant sources of uncertainty are considered within the probabilistic model of the systems (e.g. electric and thermal load variability, wind speed variability). Linkage functions are specifically designed to couple the heating system to the electrical grid and a Monte Carlo Optimal-Power-Flow (MC-OPF) simulator is employed to assess the system reliability and cost. A variance-based sensitivity analysis method is adopted to reveal the effect of different generators type, size and location on the system cost and reliability. To conclude, a multi-objective optimisation method is adopted to find a set of good investments strategies, i.e. the optimal DGs expansion plan which minimises both the system cost and unreliability index.

The rest of this paper is organised as follows: Section II introduces the probabilistic model for the weather conditions, the DGs and the heat power electric power coupling. The MC-OPF simulator system reliability and costs are presented in Section III. In Section IV an optimisation strategy for the allocation of heat and power generators is introduced. In Section $\mathrm{V}$ the Barry island case study is described and the simulation results are presented. Discussions and conclusions close the paper in Section VI.

\section{Stochastic Weather And Components MODELLING}

The structure of a power grid can be mathematically represented by a graph $\mathcal{G}_{e l}\left(\mathcal{N}_{e l}, \mathcal{E}_{e l}\right)$, such that $i$ are nodes within the set of electric grid nodes $\mathcal{N}_{e l}$ and $l=(i, j)$ are links (between nodes $i$ and $j$ ) within the edges set $\mathcal{E}_{e l}$ (set of electric cables). Similarly, the structure of a heat district network can be represented by a graph $\mathcal{G}_{t h}\left(\mathcal{N}_{t h}, \mathcal{E}_{t h}\right)$, main differences is that edges set is actualy a collection of pipes and that the node set contains the heat network nodes rather than electric grid busbars.

The framework here proposed is flexible and can account for the unavailability of specific components (e.g. unplanned maintenances for Heat pumps or WT). However, random failures of components are neglected for simplicity and only weather and operational variability accounted for in assessing network reliability. Random components failures will be accounted for in future extension of the work.

\section{A. Weather Model}

The weather model takes into account the variability of the wind speed, solar irradiance on the Earth surface and external air temperature. For simplicity, the weather condition is assumed the same over both the grids $\mathcal{G}_{e l}$ and $\mathcal{G}_{t h}$. This assumption is justified for the medium size distribution systems and heat district networks under assessment in this paper.

The wind speed $v$ is considered as a stochastic variable and it is assumed to be Rayleigh distributed. The probability distribution function (PDF) is as follows:

$$
f_{v}(v)=\frac{v}{\sigma_{v}^{2}} e^{\frac{-v^{2}}{2 \sigma_{v}^{2}}}
$$

where fitting parameter $\sigma_{v}$ can estimated using historical data for the specific geographical location in exam.

The sun radiation $s$ is assumed Beta distributed as follows [6]:

$$
f_{s}(s)=\frac{s^{a-1}(1-s)^{b-1}}{B(a, b)}
$$

where $a$ and $b$ are the distribution parameters. During night hours (e.g. $t$ from $7 \mathrm{pm}$ to $8 \mathrm{am}) s(t)$ is set to 0 .

The variability of the external air temperature $T_{\text {ext }}$ can be characterised using a normal probability distribution function i.e. $f_{T}\left(T_{e x t}\right)$. The mean $\mu_{T_{e x t}}$ and standard deviation $\sigma_{T_{e x t}}$ of the probability distribution function are fitted based on historical data from the geographical location in exam.

\section{B. Electric Power Distributed Generators}

A probabilistic model for distributed electric power generators was proposed in [6] [7] and it is reported here for completeness. Four types of electric power distributed generators are considered: Wind Turbines (WT), Photovoltaic panels (PV), Storage systems (ST) and Electric Vehicles (EV).

1) Wind Turbines: The power produced by a wind turbine depends on the (random) wind speed $v$ and is determined as follows [6]:

$$
P_{w}(v)= \begin{cases}P_{w}^{r a} \frac{v-v_{c i}}{v_{r}-v_{c i}} & \text { if } v_{c i} \leq v<v_{r} \\ P_{w}^{r a} & \text { if } v_{r} \leq v<v_{c o} \\ 0 & \text { otherwise }\end{cases}
$$


where $v_{c i}$ is the cut-in wind speed in $[\mathrm{m} / \mathrm{s}], v_{r}$ is the rated wind speed in $[\mathrm{m} / \mathrm{s}], v_{c o}$ is the cut-out wind speed in $[\mathrm{m} / \mathrm{s}]$ and $P_{w}^{r a}$ is the rated power output for the turbine in $[\mathrm{kW}]$.

2) Photovoltaic panels: The power output from PV depends on the sun radiation $s$ and on the PV parameters. The adopted model is the following [7]:

$$
\begin{gathered}
P_{p v}\left(s, T_{e x t}\right)=n_{\text {cells }} \cdot F F \cdot V \cdot I \\
T_{c}\left(s, T_{e x t}\right)=T_{e x t}+s(t) \frac{N_{o t}-20}{0.8} \\
I(s)=s(t) \cdot\left(I_{s c}+k_{i}\left(T_{c}-25\right)\right) \\
V=V_{o c}+k_{v} \cdot T_{c} \\
F F=\left(\frac{V_{M P P} \cdot I_{M P P}}{V_{o c} \cdot I_{s c}}\right)
\end{gathered}
$$

where $T_{\text {ext }}$ is the external air temperature in $\left[{ }^{\circ} \mathrm{C}\right], P_{p v}$ is the PV power output in $[\mathrm{kW}], n_{\text {cells }}$ is its number of cells, $I_{s c}$ short circuit current in [A], $k_{i}$ current temperature coefficient in $\left[\mathrm{mA} /{ }^{\circ} \mathrm{C}\right], V_{o c}$ open circuit voltage in $[\mathrm{V}], k_{v}$ voltage temperature coefficient in $\left[\mathrm{mV} /{ }^{\circ} \mathrm{C}\right] . V_{M P P}, I_{M P P}$ and $T_{c}$ are the voltage at maximum power $[\mathrm{V}]$, current at maximum power $[\mathrm{A}]$ and the cell temperature $\left[{ }^{\circ} \mathrm{C}\right]$, respectively.

3) Storage Systems and Electric Vehicles: Storage systems and, similarly, electric vehicles can inject or withdraw electric power from the network. Three EVs operating states have been considered: the vehicle to grid (V2G), the grid to vehicle $(\mathrm{G} 2 \mathrm{~V})$ and the disconnected operative states [23]. The discrete probability mass for EVs operative state $f(t, o p)$ is defined as follows [24]:

$$
f(t, o p)=\left\{\begin{array}{l}
p_{V 2 G}(t) \text { if } o p=V 2 G \\
p_{G 2 V}(t) \text { if } o p=G 2 V \\
p_{d c n}(t) \text { if } o p=\text { disconnected }
\end{array}\right.
$$

where $o p$ is an EV operative state, $p_{V 2 G}(t)$ is the V2G operative state probability at time $t, p_{G 2 V}(t)$ is the $\mathrm{G} 2 \mathrm{~V}$ operative state probability at time $t, p_{d c n}(t)$ is the probability of $\mathrm{EV}$ disconnected at time $t$ (for the probability mass values the reader is reminded to [6]) or [7]. The power injected or demanded by EVs, $P_{e v}(o p)$, is equal to plus or minus the rated power $P_{E V}^{r a}[\mathrm{~kW}]$ if the vehicle are in the discharging or charging states, respectively. If the random operative state result disconnected, $P_{e v}(o p)$ is set to 0 .

The state-of-charge $\left(E_{s t}\right)$ for a package of storage devices is assumed uniformly distributed between 0 and the maximum package capacity and, for simplicity, only discharge operative state is considered. The PDF is defined follows [7]:

$$
\begin{gathered}
f_{s t}\left(E_{s t}\right)= \begin{cases}\frac{1}{E_{S} \cdot M_{s}} & \text { if } \quad 0 \leq E_{s t} \leq E_{S} \cdot M_{s} \\
0 & \text { otherwise }\end{cases} \\
t_{r}^{\prime}=\frac{E_{s t}}{P_{s t}^{r a}} ; P_{s t}\left(t_{r}\right)=P_{s t}^{r a} \forall t_{r} \in\left[0, t_{r}^{\prime}\right]
\end{gathered}
$$

where $E_{S}$ is the specific energy of the active chemical in $[\mathrm{kJ} / \mathrm{kg}], M_{s}$ is the total mass of the active chemical in the battery $[\mathrm{kg}], P_{s t}^{r a}$ is the rated power in $[\mathrm{kW}]$ and $t_{r}^{\prime}$ is the discharging time interval $[\mathrm{h}]$.

\section{Heat Pumps}

The thermal power output $P_{H P}$ of an air-to-water mono compressor On-Off HP depends on the external air temperature as follows [18]:

$$
P_{H P}\left(T_{e x t}\right)=a_{1} \cdot T_{e x t}+b_{1} \cdot T_{e x t}^{2}+c_{1} \cdot T_{w}
$$

Similarly, the HP's coefficient of performance at full load $\left(C O P_{D C}\right)$ depends on $T_{e x t}$ and a quadratic model is adopted [18]:

$$
C O P_{D C}\left(T_{e x t}\right)=a_{2} \cdot T_{e x t}+b_{2} \cdot T_{e x t}^{2}+c_{2} \cdot T_{w}
$$

where $T_{w}$ is the hot water temperature provided to the thermal load and the regression coefficients $a_{1}, a_{2}, b_{1}, b_{2}, c_{1}, c_{2}$ are constant for a given heat pump and water temperature $T_{w}$. In the proposed model, $T_{w}$ is assumed to be $35{ }^{\circ} \mathrm{C}$ and constant, this is a realistic assumption when the HP is coupled to a radiant floor heating loop during the heating season [18]. The HP temperature operating limit $(T O L)$ depends on the specific heat pump. For the On-Off HP analysed in this work $T O L$ is equal to $-10{ }^{\circ} \mathrm{C}$ and the heat limit external temperature is assumed to be $+16{ }^{\circ} \mathrm{C}$. Thus, if $T_{\text {ext }}>+16^{\circ} \mathrm{C}$ and $T_{\text {ext }}<-10^{\circ} \mathrm{C} P_{H P}$ is set to 0 .

\section{Electric and Heat Power Loads}

The stochastic component of the electric power demanded at node $i$ and time $t$ can be characterised by a normal probability distribution as follows [7]:

$$
f\left(P_{d, e l, t, i}\right)=\frac{1}{\sqrt{2 \pi \sigma_{e l, i}(t)}} e^{-\frac{\left(P_{d, e l, t, i}-\mu_{e l, i}(t)\right)^{2}}{2 \sigma_{e l, i}(t)^{2}}}
$$

Similarly, the thermal power demanded at node $i$ and time $t$ is characterised as follows:

$$
f\left(L_{t h, t, i}\right)=\frac{1}{\sqrt{2 \pi \sigma_{t h, i}(t)}} e^{-\frac{\left(P_{d, t h, t, i}-\mu_{t h, i}(t)\right)^{2}}{2 \sigma_{t h, i}(t)^{2}}}
$$

where $\sigma_{e l, i}(t), \mu_{e l, i}(t), \sigma_{t h, i}(t)$ and $\mu_{t h, i}(t)$ are the historically fitted parameters of the probability distribution at time $t$ of the day (see [6] for further details).

\section{E. Heat and Electric Power Coupling}

The relationship between the thermal power output $\left(P_{H P}\right)$ of an heat pump is related to the electrical power demanded by the pump $\left(L_{H P, e l}\right)$ through the following relation:

$$
L_{H P, e l}=\frac{P_{H P}}{C O P_{P L}}
$$

where $C O P_{P L}$ is the coefficient of performance of the pump at partial loads. The $C O P_{P L}$ takes into account the losses linked to the on-off conditions when the pump is operated at partial regime and can be obtained by weighting the $C O P_{D C}$ 
for a function of the thermal load, the HP power output and a degradation coefficient (see [18] for further details).

In this model, when the thermal power demand exceeds the HP production in $i$, that is $P_{H P, i}<L_{t h, i}$, the residual heat demand in the node $i$ is sattisfied by a back-up heating system. For modelling simplicity only electric back-up systems are considered in this work. If the heat power demand exeeds the production the electric power required by the back-up system is computed as follows [18]:

$$
L_{B U, e l}(t)=L_{t h}(t)-P_{H P}(t)
$$

where index $i$ has been dropped for ease of notation. The aggregated electric power demand at each node is simply obtained as follows:

$$
L_{e l}(t)=P_{d, e l}(t)+L_{H T, e l}(t)+L_{B U, e l}(t)
$$

where $P_{d, e l}(t)$ is the random component of the electric power demanded by node $i$ at time $t$.

\section{Monte Carlo Optimal Power Flow AND RELIABILITY INDEX}

In this work, a Monte Carlo Optimal-Power-Flow [6] is employed to evaluate the effect of uncertainty over the system. The MC-OPF procedure is summarised by the following 5 steps:

1 Initialisation: Provide DGs size, type and location. Input number of Monte Carlo runs $\left(N_{M C}\right)$ and parameters of the stochastic model.

2 Sampling: Random sample the uncertain weather variables $\left(s, v, T_{e x t}\right)$, the time of the day $\left(t_{d}\right)$ and the grids components operative states $\left(o p(t), E_{s t}, L_{t h}(t)\right.$, $\left.L_{e l}(t)\right)$ from the stochastic model.

3 Loads/production: The weather conditions are used to compute the available power from renewable generators (see Section II-D and II-C). The coupling equations are solved and electric load increased by the electric power demanded by HPs and back-up systems.

4 Grid Analysis: The electric load and the DGs power outputs are forwarded to an optimal power flow (OPF) solver in $\mathrm{Eq} 8$. Outputs are the minimum operative cost for the grid $C_{O, i}^{\min }$ and an indicator of the system reliability and the Energy-not-Supplied ( $E N S$ ) computed as follows:

$$
E N S=\sum_{t=1}^{T_{h}} \sum_{j \in \mathcal{N}_{e l}} L_{c u t, j, t} \cdot T_{h}
$$

where $L_{c u t, j, t}$ is the load curtailed at node $j$ at time $t$ and $T_{h}$ is the simulation time.

5 Collect Results: Steps 2, 3 and 4 are repeated $N_{M C}$ times. The probability density function of the ENS and for a global cost of the system are obtained and the output expectations are computed as follow:

$$
\begin{gathered}
\mathbb{E}[E N S]=\sum_{i=1}^{N_{M C}} E N S_{i} / N_{M C} \\
\mathbb{E}\left[C_{g l b}\right]=\sum_{i=1}^{N_{M C}}\left(C_{O, i}^{m i n}+C_{i n v}-C_{i n c, i}\right) / N_{M C}
\end{gathered}
$$

where $C_{i n v}$ is the cost of the investment in distributed generators, $C_{i n c, i}$ is a gain due to the incentives available for producing power with renewable sources.

Clearly, the cost $C_{i n v}$ depends only on the initial investment in distributed generators (types and sizes) and it does not depend on the randomised scenario (no $i$ subscript). On the other hand, the incentive cost and operative cost depend on the randomised amount of available renewable power generation obtained within each scenario. The investment cost and incentive gain are computed as follows:

$$
\begin{gathered}
C_{i n v}=\sum_{J}\left(N_{J} \cdot C_{i n v, J} / T_{i n v, J}\right) \\
C_{i n c, i}=\sum_{J} I n c_{J} \cdot P_{g, J}
\end{gathered}
$$

where $N_{J}$ is the number of generators of type $J$ installed within the system, $C_{i n v, J}$ is the investment cost for the generator type $J$ and the total initial investment cost is prorated hourly over the lifetime $T_{i n v, J}$, assumed here to be 10 years for each generator type $J$ in the set $\{P V, W T, E V, S T, H P\} \quad[25] . \quad P_{g, J}$ is the total power produced by generators type $J$ within the simulation $i$. Inc $c_{J}$ are the available incentives for producing a unit of power with $J$. In $c_{J}$ is assumed here to be $2.61[\mathrm{p} / \mathrm{kWh}$ ] for renewable air source heat power [26] and $2.4[\mathrm{p} / \mathrm{kWh}$ ] plus the price of the electricity (computed as in [6]) which is saved thanks to the renewable production.

The optimal power flow solves the economic dispatch problem for the grid, i.e. it schedules the power produced by the generators so that the operational cost is minimised. In this optimal power flow formulation loads can be curtailed [6], this is done if operational or physical constraints (e.g. line thermal limits, generators capacity, etc.) cannot be fulfilled otherwise. Mathematically, the minimization problem is defined as follows [27]:

$$
C_{O, i}^{\min }=\min _{P_{g}, L_{c u t}} f\left(P_{g}, L_{c u t}\right)
$$

where $C_{O, i}^{\min }$ is the minimum total operative cost for the power grid. The cost function $f$ depends on the power produced and the load curtailed $L_{c u t}$ and has to be minimised when subject to power generation constraints, line flow and nodal active power balance constraints:

$$
f\left(P_{g}, L_{c u t}\right)=\sum_{g=1}^{N_{g}} C_{g} \cdot P_{g}+\sum_{i=1}^{N_{l}} C_{c u t} \cdot L_{c u t, i}
$$




$$
\begin{gathered}
\underline{P}_{g} \leq P_{g} \leq \bar{P}_{g} \quad \forall g=1, . ., N_{g} \\
-\bar{P}_{i, j} \leq B_{i, j} \theta_{i, j} \leq \bar{P}_{i, j} \quad \forall \quad(i, j) \in \mathcal{E}_{e l} \\
\sum_{g \in i} P_{g}+\sum_{j \neq i} B_{i, j} \theta_{i, j}+L_{c u t, i}=L_{e l, i} \quad \forall i \in \mathcal{N}_{e l}
\end{gathered}
$$

where $C_{g}$ and $C_{c u t}$ are the cost per-unit of power for the generators and the load curtailed, respectively. $N_{g}$ is the total number of electric power generators allocated within the electric grid, including both DGs and main supply generators. $\underline{P}_{g}$ and $\bar{P}_{g}$ are the minimum and maximum active power output of the generator $g$ and $L_{e l, i}$ is the aggregated electric load demanded at the power grid node $i . \bar{P}_{i, j}$ and $B_{i, j}$ are the line $(i, j)$ flow limit and susceptance, respectively. The voltage phase difference $\theta_{i, j}=\theta_{i}-\theta_{j}$ is the difference between voltage phases at nodes $i$ and $j$.

\section{Multi Objective Optimisation: Optimal Power SOURCES AllocAtion}

The goal of the optimisation analysis is to identify an optimal investment plan on distributed generators (i.e. optimal HPs, WTs, PVs, STs and EVs sizes and positions) which minimises both the $E[E N S]$ and $E\left[C_{g l b}\right]$. According to earlier works [6], the two objectives appear to be concurrent and, thus, no global optimum can be obtained (i.e. a solution minimising both the system unreliability and the cost). The optimisation problem is fairly complex due to the high dimensionality of the design space (number of DGs types and nodes) and due to the highly non-linear behaviour of the grids and components, making its analytical solution not treatable. For this reason, the Non-Dominated Sorted Genetic Algorithm version two (NSGA-II) has been employed. The algorithm allows a set of good solutions to be obtained in a single run (i.e. moving toward the Pareto's front) and it has been proven to be very effective through the incorporation of elitism in the search. Furthermore, it has no need for the sharing parameter to be chosen a priori [7] which is a clear advantage compared to previous NSGA versions.

The optimisation procedure can be summarised as follows: First, the number of generations $\left(N_{g e n}\right)$, the population size $N_{\text {pop }}$, the number of MC-OPF runs are selected. A population of chromosomes (i.e. decision variables) is randomly generated and constrained between lower and an upper limit which is fixed based on practical considerations (e.g. maximum generator size or modularity constraints). The information on the generators size and position is stored within each chromosome as Chrom $=[\mathbf{P V}, \mathbf{S T}, \mathbf{W T}, \mathbf{E V}, \mathbf{H P}]$ where, for instance, $\mathbf{P V}$ and $\mathbf{H P}$ are $\left[1 \times\left|\mathcal{N}_{e l}\right|\right]$ and $\left[1 \times\left|\mathcal{N}_{t h}\right|\right]$ vectors, respectively. The $i^{t h}$ value for PV (or HP) is the number of PVs (or HPs) allocated within the $i^{\text {th }}$ electric (or thermal) grid node. To conclude the initialization phase, each chromosome Chrom is forwarded to the MC-OPF (see Section III, and objectives $\left(\mathbb{E}[E N S]\right.$ and $\mathbb{E}\left[C_{g l b}\right]$ ) evaluated as explained in Section II-C. In the following steps, the evolutionary routine is repeated as follows. First, the

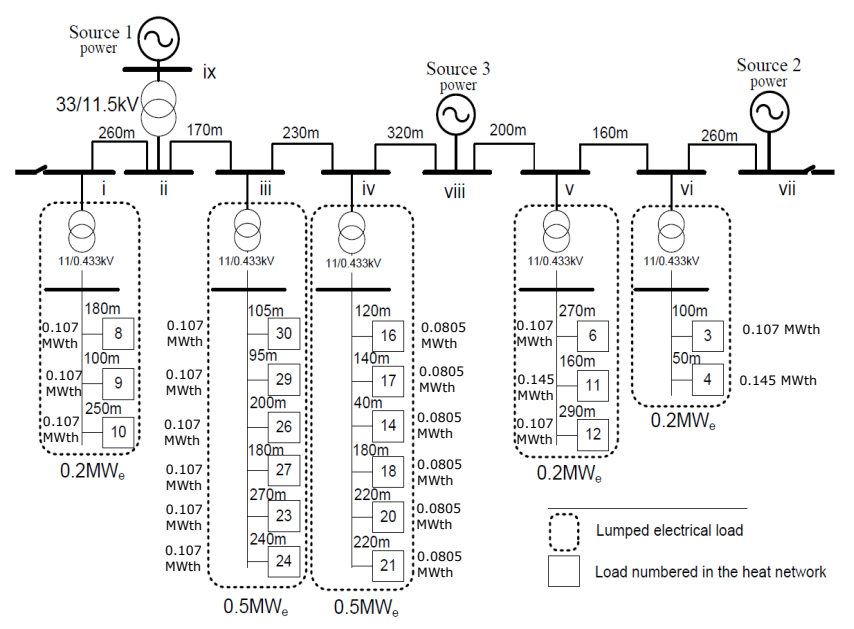

Fig. 1. The Barry island power grid and heat network (modified from $|2|$ ). In the figure are displayed links lengths, main electric generators positions and the reference magnitude for electric and thermal loads at each node.

chromosomes are sorted and ranked based on non-domination and crowding distance criteria. Then, the chromosomes with the higher score are selected and the binary crossover and polynomial mutation are performed to generate the offspring population. Each offspring then evaluated using the described MC-OPF and a fitness score is obtained. The offspring population is mixed (set union) with the previous generation of chromosomes. The chromosomes are newly sorted based on non-domination and crowding distance and the $N_{\text {pop }}$ bests are selected and the other discarded. The evolutionary routine is repeated until the predefined number of generation $N_{g e n}$ is reached.

\section{CASE Study: The BARry Island Network}

The Barry island combined heat-electric system [2] is selected to test the framework. The layout for the multienergy system is presented in Fig,1. The heat district network is composed of 32 nodes of which 20 are heat demand nodes (i.e. aggregation of buildings' heat loads). Differently from Fig 1] and accordingly to [2], an additional heat power demand is considered in node 7 and connected to the first electric busbar ( $i$ in Fig 1 ). The power grid is composed of 8 bus bars, 3 main generator sources in nodes 2,8 and 7 and 5 electric loads, lumped from the heat network as displayed in Fig 1. The 7 electric cables current rating is 400 [A] and resistances and reactances are $R=0.164$ and $X=0.08$ $[\Omega / \mathrm{km}]$, respectively. For simplicity, the heat district network pipes are neglected and only its nodes are considered in this analysis. For further details on the system, the reader is referred to [2].

The reference thermal and electrical power loads are reported within Fig 1, adapted from [2]. Generally speaking, changes in the load demand display both spatial and temporal 
TABLE I

THE DGS' MODEL DATA AND THE PARAMETERS OF THE STOCHASTIC WEATHER MODEL.

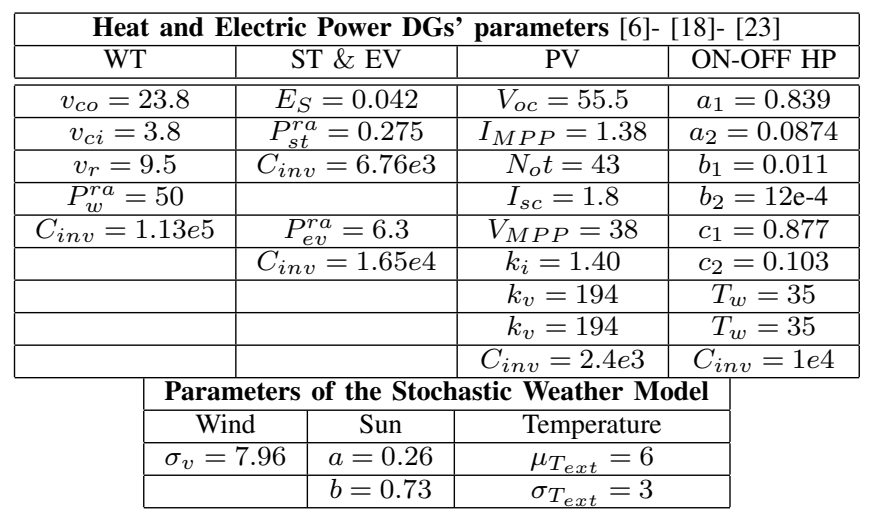

correlations, e.g. residential nodes will demand less power during night hours. The power load is assumed distributed as a normal at each grid's node and at each hour of the day, mean values and standard deviations are assumed to be a percentage of the reference loads in Fig 1 . Examples of thermal power demanded and electrical power load samples over 10 days simulation are presented in the upper and lower panel in Fig.2. respectively. The parameters of the weather stochastic model, DGs and heat pumps are reported in Table I.

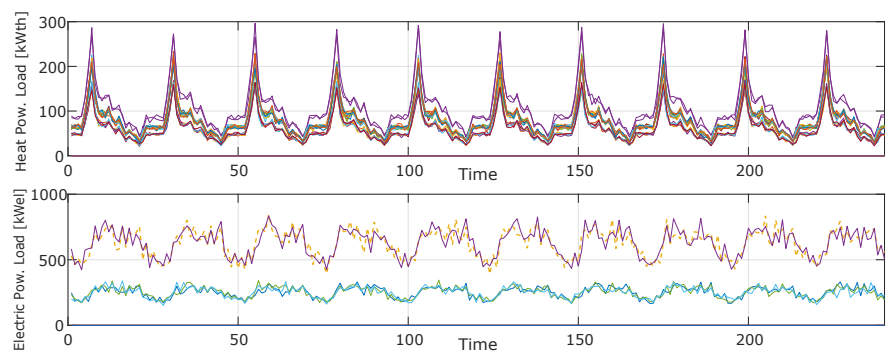

Fig. 2. An examples of random realisation of thermal power and nodal electric power demanded at each node over a simulation time of 240 hours.

\section{A. Results of the Stochastic Analysis}

The uncertainty in the system reliability index and cost is quantified using the Monte Carlo algorithm presented in Section III and 4 cases have been analysed. In the first case $(\mathrm{C}-1)$, the original network is tested (neither heat nor electric power generators have been allocated). For the second investment case (C-2), the electric grid invests on distributed electric power generators (5 PVs 5 STs and $1 \mathrm{WT}$ and $1 \mathrm{EV}$ in each node) but not on heat power generators. Case 3 (C-3) accounts for an investment on both electric and heat power generators (same as $\mathrm{C}-2$ but 1 heat pump is allocated within each node of the heat district network). For case 4 (C-4) only heat pumps have been installed. The 4 different cases are summarised using a simple vector notation as follows (see Section IV]:

\section{C-1 $[\mathbf{P V}, \mathbf{S T}, \mathbf{W T}, \mathbf{E V}, \mathbf{H P}]=[\mathbf{0}, \mathbf{0}, \mathbf{0}, \mathbf{0}, \mathbf{0}]$ \\ C-2 $[$ PV, ST, WT, EV, HP $]=[\mathbf{5}, \mathbf{5}, \mathbf{1}, \mathbf{1}, \mathbf{0}]$ \\ C-3 $[$ PV, ST, WT, EV, HP $]=[\mathbf{5}, \mathbf{5}, \mathbf{1}, \mathbf{1}, \mathbf{1}]$ \\ C-4 $[\mathbf{P V}, \mathbf{S T}, \mathbf{W T}, \mathbf{E V}, \mathbf{H P}]=[\mathbf{0}, \mathbf{0}, \mathbf{0}, \mathbf{0}, \mathbf{1}]$}

The MC-OPF run number was set to 3000 and the results of each run are the probability distribution function for the $E N S$ and $C_{g l b}$. The convergence of the method is displayed in Fig 3 for the 4 considered cases. The analysis confirms that the system reliability and the cost are concurrent objectives within the proposed model. In fact, a case with no investment (C-1 in blue dotted lines) produced the lower expected cost and lower grids reliability (i.e. higher $\mathbb{E}[E N S]$ ) whilst the case with a significant investment (C-3 in dashed yellow lines) resulted in the higher reliability but also higher $\mathbb{E}\left[C_{g l b}\right]$. On the other hand, C-2 and C-4 resulted in a compromise investment, i.e. they improved less the system reliability but for a moderate cost. The results pointed out that a combined investment on HP and electric power generators can provide a significant improvement in the system reliability. The expected values for the system cost and reliability, the coefficients of variations $\left(C_{V}\right)$ and $95^{\text {th }}$ percentiles $\left(p_{95}\right)$ for the 4 analysed cases are reported in TableII It can be seen that, although higher expected reliability can be obtained by investing on DGs, the $C_{V}$ increases when renewable and distributed generators are allocated within the network. This indicates an inherently higher variability of grids systems when renewable energy sources are adopted which might also indicate a higher risk of hazardous, unexpected low probability scenarios.

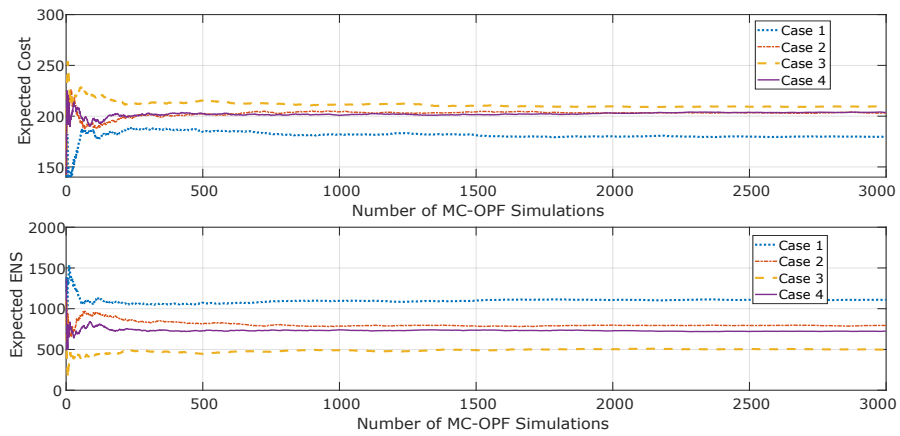

Fig. 3. The $\mathbb{E}[E N S]$ and $\mathbb{E}\left[C_{g l b}\right]$ for increasing number of MC-OPF runs and the 4 considered investment cases.

TABLE II

THE RESUlTS OF THE 4 INVESTMENT CASES ON HEAT AND ELECTRIC POWER GENERATORS

\begin{tabular}{|c|c|c|c|c|}
\hline Case & $\mathbf{C - 1}$ & $\mathbf{C - 2}$ & $\mathbf{C - 3}$ & $\mathbf{C - 4}$ \\
\hline $\mathbb{E}[E N S]$ & 1110.4 & 793.7 & 498 & 723.9 \\
\hline$C_{V}[E N S]$ & 0.59 & 0.85 & 1.18 & 0.87 \\
\hline$p_{95}[E N S]$ & 2286 & 2178 & 1617 & 1779 \\
\hline $\mathbb{E}\left[C_{g l b}\right]$ & 179.5 & 203.2 & 209.7 & 203.8 \\
\hline$C_{V}\left[C_{g l b}\right]$ & 0.45 & 0.39 & 0.31 & 0.37 \\
\hline$p_{95}\left[C_{g l b}\right]$ & 291 & 302 & 289 & 296 \\
\hline
\end{tabular}




\section{B. Optimisation and Sensitivity Results}

The sensitivity of the expected system cost and expected unreliability to generators sizes and positions has been assessed by adopting a variance-based sensitivity method [28]. This method allows first order Sobol's sensitivity indices to be obtained (i.e. relative changes in outputs variances fixing decision variables one-at-a-time) and with the advantage of a relatively small computational cost. A Latin Hypercube method [29] was used to sample 25000 realisations from the design space (i.e. Chrom vectors) and each realisation is a vector of 64 elements. The first 32 elements are electric DGs numbers ( 4 for each of the 8 electric nodes) whilst the last 32 elements are the number of HPs which have been allocated to the existing heat district network nodes. The number of PVs and STs generators in each electric node can vary between 0 and 10 whilst for other generators (wind turbines, electric vehicles and air source heat pumps) it can vary between 0 and 2. The expected cost and expected ENS have been estimated using the Monte Carlo OPF method for each realisation. The first order Sobol's indices, i.e. $S_{i}=\frac{\operatorname{Var}\left[\mathbb{E}\left[y \mid x_{i}\right]\right]}{\operatorname{Var}[y]}$, have been obtained for each design variable $x_{i}$ and each output $y$. In this analysis, the considered outputs $y$ are the expected $E N S$ and expected $C_{g l b}$ and the sensitivity results are presented in top and bottom panel of Fig 4 respectively. It can be observed a sensitivity of the objective to specific technologies. In fact, the allocation of wind turbines affects the most the system reliability followed by heat pumps in the heat district network. On the other hand, other generators seem less influencing the system cost and reliability, probably due to the smaller power output capacity and the limited modularity allowed within this analysis (e.g. max $10 \mathrm{PVs}$ and $10 \mathrm{STs}$ ). In addition to a technological sensitivity, we can also observe a nodal sensitivity (i.e. sensitivity to a specific nodal allocation of heat pumps). Consider for instance nodes 1, 2 or 3. Introducing HPs in those nodes does not affect the system reliability and this can be explained considering the topology of the heat district network. In $\mathcal{G}_{t h}$, the nodes 1, 2 and 3 are not load-carrying nodes and therefore allocate there HPs does not provide any benefit for the system (also because pipes are neglected for this analysis).

The NSGA-II optimisation procedure starts by selecting $N_{\text {pop }}, N_{\text {gen }}$ and $N_{M C}$ set to 500,50 and 2000, respectively. Each chromosome decision vector is, in this case, a list of 64 integers which indicate the amount and type of distributed generators allocated in each node. A maximum of 10 photovoltaic generators and storage systems are assumed in each bus bar, whilst up to 2 wind turbines, electric vehicles and air source heat pumps can be allocated. The fitness of the best chromosomes evaluated within the last generation are displayed in Fig 5 The y-axis contains the Energy-notsupplied expectation and the $y$-axis contains the global cost expectation; 3 solutions have been selected and displayed with red diamond markers. A 'best reliable' solution with higher reliability, a 'best cheap' solution having the lower cost (but

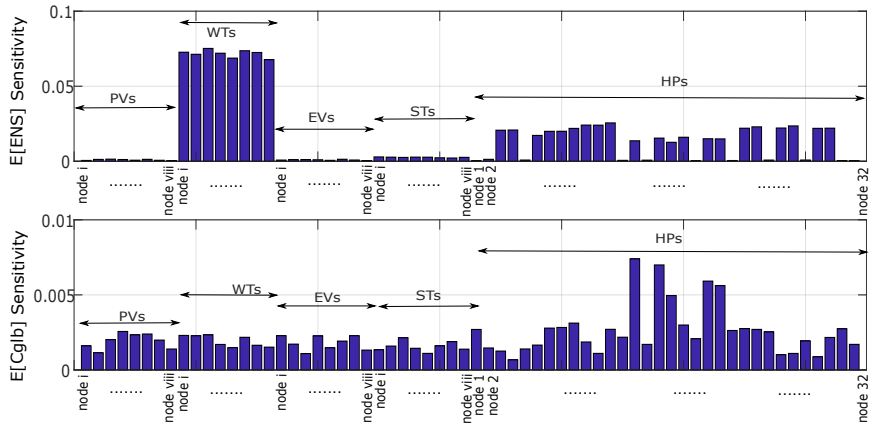

Fig. 4. The first order sensitivity indices $S_{i}$ for the expected $E N S$ (top panel) and expected cost (bottom panel).

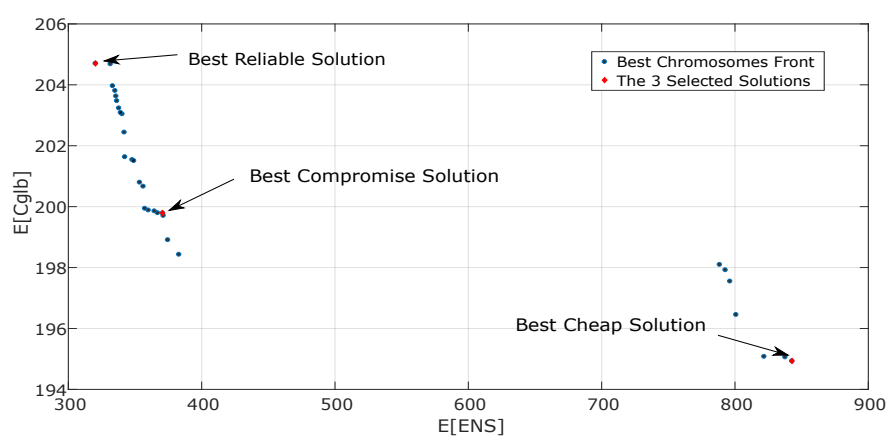

Fig. 5. The 3 best chromosomes selected among the best front in the generation of the NSGA-II procedure.

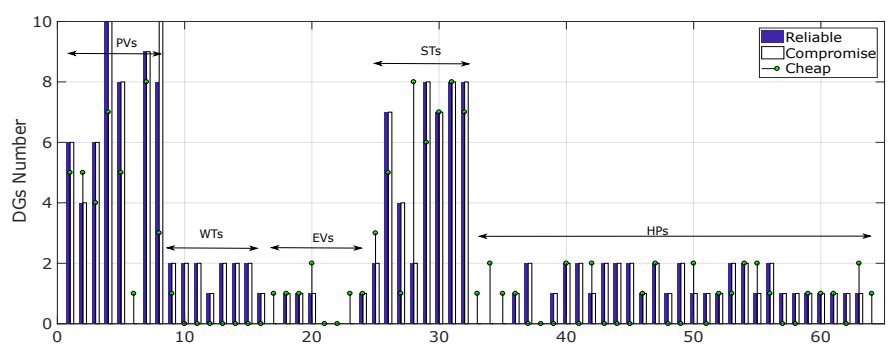

Fig. 6. The renewable generator number, type and position for the 3 best solutions selected.

more unreliable) and a third with a compromise cost and reliability (see Fig 5 ). The generator sizes per each grids' node are presented in Fig 6 and statistical results retailed from the $\mathrm{MC}-\mathrm{OPF}$ are presented in Table III.

\section{DisCUSSION AND CONCLUSION}

This paper presented a stochastic framework for simulations and analysis of coupled electric and heat power networks. A Monte Carlo optimal power flow simulator is used to analyse different investment scenarios in heat and electric power generation technologies. A probabilistic model to describe weather conditions and grid's operations variability has been proposed. This allows random realistic scenarios to be 
TABLE III

LOW COST INVESTMENT, HIGH RELIABILITY INVESTMENT AND THE COMPROMISE SOLUTION FOR THE BARRY ISLAND NETWORK

\begin{tabular}{|c|c|c|c|}
\hline Solution & Reliable & Compromise & Cheap \\
\hline $\mathbb{E}[E N S]$ & 320 & 370 & 842 \\
\hline $\mathbb{E}\left[C_{g l b}\right]$ & 205 & 200 & 195 \\
\hline$C_{V}[E N S]$ & 1.55 & 1.46 & 0.79 \\
\hline$p_{95}[E N S]$ & 1302 & 1578 & 2520 \\
\hline$C_{V}\left[C_{g l b}\right]$ & 0.27 & 0.29 & 0.42 \\
\hline$p_{95}\left[C_{g l b}\right]$ & 287.1 & 284.3 & 301.1 \\
\hline
\end{tabular}

generated by the simulator and uncertainty quantified in the grids reliability and global cost. The proposed framework has been applied to a representative case study: The Barry Island power-heat network. Four different investment scenarios have been analysed. Results show that a combined investment on renewable heat and electric power generators provides greater benefits for the system in terms of reliability, and for a moderate cost due to available incentives. Sensitivity analysis pointed out that installation of wind turbines and heat pumps are affecting most the system reliability and that the load-carrying nodes were the only relevant nodes for the system outputs. To conclude, an optimisation strategy was employed to obtain a set of best investments on renewable heat and electric sources. The optimisation provides a set of good investments, in terms of sizing and positioning of renewable energy sources, which minimises both system unreliability and global cost. The high dimensionality and the stochastic nature of the fitness function have been successfully addressed by the proposed method.

\section{ACKNOWLEDGMENT}

The authors would like to acknowledge the gracious support of this work through the EPSRC and ESRC Centre for Doctoral Training on Quantification and Management of Risk \& Uncertainty in Complex Systems \& Environments Grant number (EP/L015927/1)

\section{REFERENCES}

[1] R. Bayindir, I. Colak, G. Fulli, and K. Demirtas, "Smart grid technologies and applications," Renewable and Sustainable Energy Reviews, vol. 66, pp. $499-516,2016$

[2] X. Liu, J. Wu, N. Jenkins, and A. Bagdanavicius, "Combined analysis of electricity and heat networks," Applied Energy, vol. 162, pp. 1238 $1250,2016$.

[3] P. Adhvaryyu, P. Chattopadhyay, and A. Bhattacharya, "Dynamic optimal power flow of combined heat and power system with valve-point effect using krill herd algorithm," Energy, vol. 127, pp. 756 - 767, 2017.

[4] P. Li, N. Nord, I. S. Ertesvåg, Z. Ge, Z. Yang, and Y. Yang, "Integrated multiscale simulation of combined heat and power based district heating system," Energy Conversion and Management, vol. 106, pp. 337 - 354, 2015.

[5] P. Mancarella, "Mes (multi-energy systems): An overview of concepts and evaluation models," Energy, vol. 65, pp. 1 - 17, 2014.

[6] R. Mena, M. Hennebel, Y.-F. Li, and E. Zio, "A multi-objective optimization framework for risk-controlled integration of renewable generation into electric power systems," Energy, vol. 106, pp. 712 - 727, 2016.

[7] R. Rocchetta, Y. Li, and E. Zio, "Risk assessment and risk-cost optimization of distributed power generation systems considering extreme weather conditions," Reliability Engineering \& System Safety, vol. 136, no. 0 , pp. $47-61,2015$.
[8] C. Furlan and C. Mortarino, "Forecasting the impact of renewable energies in competition with non-renewable sources," Renewable and Sustainable Energy Reviews, pp. -, 2017.

[9] K. P. Kumar and B. Saravanan, "Recent techniques to model uncertainties in power generation from renewable energy sources and loads in microgrids - a review," Renewable and Sustainable Energy Reviews, vol. 71, pp. 348 - 358, 2017.

[10] E. Patelli, Handbook of Uncertainty Quantification. Cham: Springer International Publishing, 2016, ch. COSSAN: A Multidisciplinary Software Suite for Uncertainty Quantification and Risk Management, pp. $1-69$.

[11] E. Patelli, D. Alvarez, M. Broggi, and M. De Angelis, "Uncertainty management in multidisciplinary design of critical safety systems," Journal of Aerospace Information Systems, vol. 12, no. 1, pp. 140-169, 2015.

[12] H. Karami, M. Sanjari, H. Gooi, G. Gharehpetian, and J. Guerrero, "Stochastic analysis of residential micro combined heat and power system," Energy Conversion and Management, vol. 138, pp. 190 - 198, 2017.

[13] E. Patelli, M. Broggi, M. Angelis, and M. Beer, "Opencossan: An efficient open tool for dealing with epistemic and aleatory uncertainties," in Vulnerability, Uncertainty, and Risk. American Society of Civil Engineers, 2014, pp. 2564-2573.

[14] U. Berardi, "A cross-country comparison of the building energy consumptions and their trends," Resources, Conservation and Recycling, vol. 123 , pp. $230-241,2017$.

[15] C. Zeng, S. Liu, and A. Shukla, "A review on the air-to-air heat and mass exchanger technologies for building applications," Renewable and Sustainable Energy Reviews, vol. 75, pp. 753 - 774, 2017.

[16] S. Charlesworth, A. Faraj-Llyod, and S. Coupe, "Renewable energy combined with sustainable drainage: Ground source heat and pervious paving," Renewable and Sustainable Energy Reviews, vol. 68, Part 2, pp. $912-919,2017$.

[17] R. S. Adhikari, M. Buzzetti, and S. Magelli, "Solar photovoltaic and thermal systems for electricity generation, space heating and domestic hot water in a residential building," in 2011 International Conference on Clean Electrical Power (ICCEP), June 2011, pp. 461-465.

[18] M. Dongellini, C. Naldi, and G. L. Morini, "Seasonal performance evaluation of electric air-to-water heat pump systems," Applied Thermal Engineering, vol. 90, pp. 1072 - 1081, 2015.

[19] X. Dong, Q. Tian, and Z. Li, "Experimental investigation on heating performance of solar integrated air source heat pump," Applied Thermal Engineering, vol. 123, pp. 1013 - 1020, 2017.

[20] L. Yang, N. Tai, C. Fan, and Y. Meng, "Energy regulating and fluctuation stabilizing by air source heat pump and battery energy storage system in microgrid," Renewable Energy, vol. 95, pp. 202 - 212, 2016.

[21] M. J. M. A. Essa and L. M. Cipcigan, "Integration of renewable resources into low voltage grids stochastically," in 2016 IEEE International Energy Conference (ENERGYCON), April 2016, pp. 1-5.

[22] E. A. M. Cesena, T. Capuder, and P. Mancarella, "Flexible distributed multienergy generation system expansion planning under uncertainty," IEEE Transactions on Smart Grid, vol. 7, no. 1, pp. 348-357, Jan 2016.

[23] K. Clement-Nyns, E. Haesen, and J. Driesen, "The impact of vehicle-togrid on the distribution grid," Electric Power Systems Research, vol. 81, no. 1, pp. $185-192,2011$.

[24] Y. Li and E. Zio, "Uncertainty analysis of the adequacy assessment model of a distributed generation system," Renewable Energy, vol. 41, pp. $235-244,2012$.

[25] R. Mena, E. Zio, and M. Hennebel, "Sensitivity analysis of a simulation model for evaluating renewable distributed generation on a power network," in 2014 International Conference on Probabilistic Methods Applied to Power Systems (PMAPS), July 2014, pp. 1-6.

[26] [Online]. Available: http://www.icax.co.uk/Renewable_Heat_Incentive. html

[27] R. Rocchetta and E. Patelli, "An efficient framework for reliability assessment of power networks installing renewable generators and subject to parametric p-box uncertainty," in Conference: The 2nd International Conference on Engineering Sciences and Technologies.

[28] E. Plischke, E. Borgonovo, and C. L. Smith, "Global sensitivity measures from given data," European Journal of Operational Research, vol. 226, no. 3 , pp. $536-550,2013$

[29] B. Tang, "Orthogonal array-based latin hypercubes," Journal of the American Statistical Association, vol. 88, no. 424, pp. 1392-1397, 1993. 